\title{
28 Research Square \\ Genetic adaptations to extreme conditions in the Middle East linked to metabolic syndrome
}

\author{
Muthukrishnan Eaaswarkhanth \\ Andre Luiz Campelo dos Santos \\ Omer Gokcumen \\ Fahd Al-Mulla \\ Thangavel Alphonse Thanaraj
}

\section{Video Abstract}

Keywords: Dasman Diabetes Institute, Kuwait population, positive selection, TNKS, metabolic traits, hypertension, metabolic syndrome, Arabian Peninsula, haplotype, homozygosity, population branch statistics, genetic diversity, PLINK, single nucleotide polymorphism, SNP, population differentiation, linkage disequilibrium, iHS, sympathetic nervous system, pleiotropic

Posted Date: October 23rd, 2020

DOI: https://doi.org/10.21203/rs.3.rs-97531/v1

License: (c) (i) This work is licensed under a Creative Commons Attribution 4.0 International License. Read Full License 


\section{Abstract}

Obesity and metabolic disorders are abnormally high in the Middle East. According to the World Health Organization, approximately $41 \%$ of all deaths in Kuwait are caused by cardiovascular disease. And after the US, Kuwait is the nation with the highest rate of obesity in the world. While overeating and an increasingly sedentary lifestyle are partially to blame, they're not the only culprits. A new study from the Dasman Diabetes Institute in Kuwait suggests that genetic adaptations once key to survival for Kuwaiti ancestors predispose today's population to debilitating disease. Traits like insulin resistance and hypertension spell trouble for modern, largely sedentary humans. But for the nomadic forbearers of today's Kuwaiti population, they offered an advantage. An active metabolism and high blood pressure stimulate the sympathetic nervous system's "fight-flight-or-freeze" response, favoring survival under the harsh desert climate of the Arabian Peninsula. In the recent study, genotyping of 583 healthy, unrelated Kuwaiti individuals showed that these genetic adaptations occur across a gene called TNKS and are associated with hypertension, obesity, and type 2 diabetes. Having three or more risk factors like these is indicative of metabolic syndrome, according to the American Heart Association. Post-oil-boom urbanization across the Arabian Peninsula has transformed the lifestyle of the people in this region. They've gone from keeping mobile to battle food scarcity and scorching temperatures to maintaining low physical activity and consuming calorie-rich foods. But their genetics have remained largely the same. That combination has likely made the adapted regions of the TNKS gene fatal for millions who call the Arabian Peninsula home. More work is needed to understand the genetic origins of metabolic disease in this area of the world. But the current study lays a solid foundation. The findings help establish that genetic adaptations once considered key to the survival of nomadic Arabic society have worn out their welcome, and could now contribute to the negative health effects experienced by their descendants. 